\title{
A Post Hoc Comparison of the Effects of Lisdexamfetamine Dimesylate and Osmotic-Release Oral System Methylphenidate on Symptoms of Attention-Deficit Hyperactivity Disorder in Children and Adolescents
}

\author{
César Soutullo - Tobias Banaschewski - Michel Lecendreux • Mats Johnson • \\ Alessandro Zuddas - Colleen Anderson - Richard Civil • Nicholas Higgins • \\ Ralph Bloomfield $\cdot$ Liza A. Squires $\cdot$ David R. Coghill
}

Published online: 26 June 2013

(c) The Author(s) 2013. This article is published with open access at Springerlink.com

\begin{abstract}
Introduction There are limited head-to-head data comparing the efficacy of long-acting amfetamine- and methylphenidate-based psychostimulants as treatments for individuals with attention-deficit hyperactivity disorder (ADHD). This post hoc analysis provides the first parallelgroup comparison of the effect of lisdexamfetamine dimesylate (lisdexamfetamine) and osmotic-release oral system methylphenidate (OROS-MPH) on symptoms of ADHD in children and adolescents.

Study Design This was a post hoc analysis of a randomized, double-blind, parallel-group, dose-optimized, placebo-controlled, phase III study.

Setting The phase III study was carried out in 48 centres across ten European countries.

Patients The phase III study enrolled children and adolescents (aged 6-17 years) who met Diagnostic and
\end{abstract}

C. Soutullo $(\square)$

Child and Adolescent Psychiatry Unit, Department of Psychiatry and Medical Psychology, University of Navarra Clinic, Pio XII, s/n, 31008 Pamplona, Spain

e-mail: csoutullo@unav.es

T. Banaschewski

Child and Adolescent Psychiatry and Psychotherapy, Central Institute of Mental Health, Medical Faculty Mannheim of the University of Heidelberg, Mannheim, Germany

\section{Lecendreux}

Paediatric Sleep Centre and National Reference Centre for Orphan Diseases: Narcolepsy, Idiopathic Hypersomnia and Kleine-Levin Syndrome, Robert-Debré University Hospital, Paris, France

M. Johnson

Child Neuropsychiatry Unit, Queen Silvia Children's Hospital,

Gothenburg, Sweden
Statistical Manual of Mental Disorders, Fourth Edition, Text Revision criteria for a primary diagnosis of ADHD and who had a baseline ADHD Rating Scale IV (ADHDRS-IV) total score of 28 or higher.

Intervention Eligible patients were randomized (1:1:1) to receive a once-daily, optimized dose of lisdexamfetamine (30, 50 or $70 \mathrm{mg} /$ day), placebo or OROS-MPH (18, 36 or $54 \mathrm{mg} /$ day) for 7 weeks.

Main Outcome Measures In this post hoc analysis, efficacy was assessed using the ADHD-RS-IV and Clinical Global Impressions-Improvement (CGI-I) scale. Responders were defined as those achieving at least a $30 \%$ reduction from baseline in ADHD-RS-IV total score and a CGI-I score of 1 (very much improved) or 2 (much improved). The proportion of patients achieving an ADHD-RS-IV total score less than or equal to the mean for their age (based on normative data) was also determined.

\author{
A. Zuddas \\ Department of Biomedical Sciences, Section of Neuroscience \\ and Clinical Pharmacology, University of Cagliari, \\ Cagliari, Italy \\ C. Anderson - R. Civil · N. Higgins · L. A. Squires \\ Shire Development LLC, Wayne, PA, USA \\ R. Bloomfield \\ Shire Pharmaceutical Development Ltd, Basingstoke, UK \\ D. R. Coghill \\ Division of Neuroscience, University of Dundee, Dundee, UK
}


Endpoint was the last on-treatment visit with a valid assessment. Safety assessments included treatment-emergent adverse events (TEAEs) and vital signs.

Results Of the 336 patients randomized, 332 were included in the safety population, 317 were included in the full analysis set and 196 completed the study. The mean (standard deviation) ADHD-RS-IV total score at baseline was 40.7 (7.31) for lisdexamfetamine, 41.0 (7.14) for placebo and 40.5 (6.72) for OROS-MPH. The least-squares (LS) mean change (standard error) in ADHD-RS-IV total score from baseline to endpoint was -24.3 (1.16) for lisdexamfetamine, -5.7 (1.13) for placebo and -18.7 (1.14) for OROS-MPH. The difference between lisdexamfetamine and OROS-MPH in LS mean change (95\% confidence interval [CI]) in ADHD-RS-IV total score from baseline to endpoint was statistically significant in favour of lisdexamfetamine (-5.6 [-8.4 to -2.7$] ; p<0.001)$. The difference between lisdexamfetamine and OROS-MPH in the percentage of patients $(95 \% \mathrm{CI})$ with a CGI-I score of 1 or 2 at endpoint was $17.4(5.0-29.8 ; p<0.05$; number needed to treat [NNT] 6), and the difference in the percentage of patients (95\% CI) achieving at least a $30 \%$ reduction in ADHD-RS-IV total score and a CGI-I score of 1 or 2 was $18.3(5.4-31.3 ; p<0.05$; NNT 6). The difference between lisdexamfetamine and OROS-MPH in the percentage of patients $(95 \% \mathrm{CI})$ with an ADHD-RS-IV total score less than or equal to the mean for their age at endpoint was $14.0(0.6-27.4 ; p=0.050)$. The overall frequency of TEAEs and the frequencies of decreased appetite, insomnia, decreased weight, nausea and anorexia TEAEs were greater in patients treated with lisdexamfetamine than in those treated with OROS-MPH, whereas headache and nasopharyngitis were more frequently reported in patients receiving OROS-MPH.

Conclusions This post hoc analysis showed that, at the doses tested, patients treated with lisdexamfetamine showed statistically significantly greater improvement in symptoms of ADHD than those receiving OROS-MPH, as assessed using the ADHD-RS-IV and CGI-I. The safety profiles of lisdexamfetamine and OROS-MPH were consistent with the known effects of stimulant medications.

\section{Introduction}

Amfetamine- and methylphenidate-based stimulants are effective pharmacological treatments for individuals with attention-deficit hyperactivity disorder (ADHD), which is one of the most common neurodevelopmental disorders among school-aged children [1-3]. These pharmacotherapies have consistently been found to reduce the symptoms of inattention, hyperactivity and/or impulsivity, as well as the functional impairments that are associated with ADHD [4, 5].
Long-acting stimulant formulations were developed to provide extended control of ADHD symptoms throughout the day [6]. Lisdexamfetamine dimesylate (lisdexamfetamine) and osmotic-release oral system methylphenidate (OROS-MPH) were both designed to facilitate once-daily dosing. Lisdexamfetamine is the first long-acting prodrug stimulant. It is metabolized primarily in the bloodstream after absorption from the gastrointestinal tract, yielding therapeutically active $d$-amfetamine [7, 8]. OROS-MPH capsules deliver the active drug, racemic methylphenidate, in the gastrointestinal tract in a biphasic manner [9]. Therapeutic benefits have been shown to persist for 13-14 h with lisdexamfetamine [10, 11] and $12.5 \mathrm{~h}$ [12] with OROS-MPH. A recent meta-analysis provided indirect evidence that stimulants based on amfetamine may have slightly greater efficacy than those based on methylphenidate in reducing symptoms of ADHD in children and adolescents [1]. However, to date, no published, parallelgroup studies have directly compared the efficacy of lisdexamfetamine and OROS-MPH.

Study SPD489-325 was a European, 7-week, phase III, randomized study that evaluated the efficacy and safety of lisdexamfetamine in children and adolescents with ADHD [13]. The study utilised a three-arm design that included a placebo control and an active reference arm, as required by the European Medicines Agency [14]. Lisdexamfetamine and the active comparator, OROS-MPH, were shown to be more effective than placebo in reducing symptoms of ADHD, as assessed using the ADHD Rating Scale IV (ADHD-RS-IV) and the Clinical Global ImpressionsImprovement scale (CGI-I) [13]. Improvements in ADHDRS-IV total score from baseline to endpoint were associated with large effect sizes for lisdexamfetamine (1.80) and OROS-MPH (1.26), indicating robust treatment responses. Although study SPD489-325 was neither planned nor powered for a primary statistical comparison between the two active treatment arms, the present post hoc analysis was conducted to compare the effect of lisdexamfetamine and OROS-MPH on symptoms of ADHD in children and adolescents.

\section{Methods}

The experimental procedures used in this randomized, double-blind, parallel-group, dose-optimized, placebocontrolled, phase III study have been described previously [13]. The study protocol (ClinicalTrials.gov ID: NCT00763971) was approved by an independent ethics committee/institutional review board and regulatory agency in each centre (as appropriate) before study initiation. The study was conducted in accordance with current international and local applicable regulations, and written 
informed consent was obtained from each participant or their legally appointed representative.

\subsection{Patients and Study Design}

The study was conducted in 48 centres across ten European countries and enrolled male and female children (aged 6-12 years) and adolescents (aged 13-17 years) who met the Diagnostic and Statistical Manual of Mental Disorders, Fourth Edition, Text Revision (DSM-IV-TR) criteria for a primary diagnosis of ADHD. Patients were required to have an investigator-rated, baseline ADHD-RS-IV total score of 28 or higher. Enrolment was managed so that adolescents (aged 13-17 years) accounted for approximately $25 \%$ of the study population.

Eligible patients completed a screening and washout period (3-42 days, depending on previous medication) and were randomized (1:1:1) to receive once-daily lisdexamfetamine, placebo or OROS-MPH. The double-blind evaluation period consisted of a 4-week dose-optimization period, followed by a 3-week dose-maintenance period, and a 1-week washout and safety follow-up.

Three doses of lisdexamfetamine $(30,50$ and $70 \mathrm{mg} /$ day) and OROS-MPH (18, 36 and $54 \mathrm{mg} /$ day) were used in this study. OROS-MPH was administered according to European regulations (maximum licensed dose, $54 \mathrm{mg}$ / day) [15]. Dosing began at approximately 07:00 $\mathrm{h}$ on the morning after completion of the baseline visit. Patients initially received lisdexamfetamine $30 \mathrm{mg} /$ day, placebo or OROS-MPH $18 \mathrm{mg} / \mathrm{day}$. If an acceptable response to treatment was not achieved, adjustments to higher doses were to be made at weekly intervals during the dose-optimization period. An acceptable response was defined as a reduction of at least $30 \%$ in ADHD-RS-IV total score from baseline and a CGI-I score of 1 (very much improved) or 2 (much improved), with tolerable adverse effects. One dose reduction was permitted during the optimization period if a patient experienced an intolerable adverse effect. Doses could not be modified after visit 3; patients unable to tolerate the study drug after visit 3 were withdrawn from the study. Patients achieving an acceptable response continued on their optimal dose for the remainder of the double-blind evaluation period.

\subsection{Efficacy Outcomes}

The primary efficacy outcome measure of SPD489-325 was the investigator-rated ADHD-RS-IV total score, which was assessed at baseline and at each weekly study visit thereafter. The key secondary efficacy outcome measure was the investigator-rated CGI-I, which was used to assess global improvement at each weekly post-baseline visit. CGI-I scores were categorized as 'improved' (CGI-I of 1 or 2) or 'not improved' (all other scores). A clinically significant response was defined a priori as at least a $30 \%$ reduction from baseline in ADHD-RS-IV total score and a CGI-I score of 1 or 2 [16]. The proportions of patients achieving an ADHD-RS-IV total score less than or equal to the mean for their age, based on normative data, were also determined.

\subsection{Safety Outcomes}

Safety outcomes were assessed for the safety population, defined as all patients who took at least one dose of study drug. Safety assessments included, but were not limited to, evaluation of treatment-emergent adverse events (TEAEs), clinical laboratory parameters, vital signs and electrocardiograms, as well as physical examinations. An adverse event was defined as treatment emergent if the event started or worsened in the period between the first dose of study drug and the third day (inclusive) following cessation of treatment. TEAEs were coded using the current version of the Medical Dictionary for Regulatory Activities (version 11.1) and summarized by system organ class, preferred term and treatment group for the number and proportion reporting the event.

\subsection{Statistical Analyses}

Although not pre-specified in the statistical analysis plan for SPD489-325, a post hoc statistical analysis was conducted to compare the effect of lisdexamfetamine and OROS-MPH on symptoms of ADHD, as assessed using the ADHD-RS-IV and CGI-I.

Efficacy outcomes were assessed for the full analysis set, defined as all patients who were randomized and took at least one dose of study drug. Patients from one site $(n=15)$ were excluded from the full analysis set as a consequence of violations of Good Clinical Practice. The change from baseline in the ADHD-RS-IV total score was analysed using an analysis of covariance (ANCOVA) model. Least squares (LS) means and $p$ values were based on type III sum of squares from the ANCOVA model for the change from baseline, including treatment group (effect of interest), country and age group (randomization blocking factors) and the corresponding baseline score (covariate). Effect sizes based on the change in ADHD-RS-IV total score from baseline were calculated as the difference in LS mean score between treatment arms, divided by the root mean square error obtained from the ANCOVA model. The number and percentage of patients categorized as 'improved' (CGI-I of 1 or 2) at each post-baseline study visit and at endpoint was summarized by treatment group, and each active treatment group compared with placebo using a Cochran-Mantel-Haenszel test stratified by 
country and age group. The percentage of patients meeting each responder criterion at endpoint was also analysed using a Cochran-Mantel-Haenszel test stratified by country and age group. The number needed to treat (NNT) was calculated as the inverse of the difference in proportions between the treatment groups. The endpoint for all outcome measures was the last on-treatment, post-baseline visit with a valid assessment.

Safety data are summarized for the safety population using descriptive statistics; no statistical tests were performed.

\section{Results}

\subsection{Patients}

Of the 336 patients who were randomized, 332 were included in the safety population (lisdexamfetamine, $n=111$; placebo, $n=110$; OROS-MPH, $n=111$ ), 317 were included in the full analysis set (lisdexamfetamine, $n=104$; placebo, $n=106$; OROS-MPH, $n=107$ ) and 196 completed the study (lisdexamfetamine, $n=80$; placebo, $n=42$; OROS-MPH, $n=74$ ). Patient demographics and baseline characteristics were similar across treatment groups (Table 1).

\subsection{Efficacy Outcomes}

At baseline, mean ADHD-RS-IV total scores (standard deviation [SD]) were similar across treatment groups (lisdexamfetamine 40.7 [7.31]; placebo 41.0 [7.14]; OROSMPH 40.5 [6.72]) [13]. The LS mean change (standard error) in ADHD-RS-IV total score from baseline to endpoint was -24.3 (1.16) for lisdexamfetamine, -5.7 (1.13) for placebo and -18.7 (1.14) for OROS-MPH [13]. The difference (active drug minus placebo) in the LS mean change in ADHD-RS-IV total score was statistically significant for lisdexamfetamine $(p<0.001$; effect size 1.80) and OROS-MPH $(p<0.001$; effect size 1.26) (Table 2) [13]. The percentage of patients $(95 \% \mathrm{CI})$ with a CGI-I score of 1 or 2 at endpoint was $78.0 \%$ (69.9-86.1) for lisdexamfetamine, $14.4 \%(7.7-21.2)$ for placebo and $60.6 \%(51.2-70.0)$ for OROS-MPH. The difference (active drug minus placebo) in the percentage of patients with a CGI-I score of 1 or 2 was statistically significant for lisdexamfetamine $(p<0.001$; NNT 2$)$ and OROS-MPH $(p<0.001$; NNT 3) (Table 2) [13].

A clinically significant response to treatment was defined a priori as at least a $30 \%$ reduction from baseline in ADHDRS-IV total score and a CGI-I score of 1 or 2. At endpoint, the percentage of patients $(95 \% \mathrm{CI})$ categorized as responders was $74.2 \%(65.5-82.9)$ for lisdexamfetamine,
Table 1 Baseline characteristics and demographic data (safety population) $)^{\mathrm{a}}$

\begin{tabular}{|c|c|c|c|}
\hline Characteristic & $\begin{array}{l}\mathrm{LDX} \\
(n=111)\end{array}$ & $\begin{array}{l}\text { Placebo } \\
(n=110)\end{array}$ & $\begin{array}{l}\text { OROS-MPH } \\
(n=111)\end{array}$ \\
\hline Age, years, mean (SD) & $10.9(2.9)$ & $11.0(2.8)$ & $10.9(2.6)$ \\
\hline Sex, male, $n(\%)$ & $87(78.4)$ & $91(82.7)$ & $90(81.1)$ \\
\hline Race, white, $n(\%)$ & $107(96.4)$ & $108(98.2)$ & $107(96.4)$ \\
\hline BMI, $\mathrm{kg} / \mathrm{m}^{2}$, mean (SD) & $19.3(3.7)$ & $19.0(3.3)$ & $19.1(3.2)$ \\
\hline 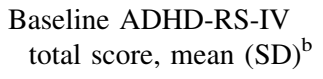 & $41.0(7.3)$ & $41.2(7.2)$ & $40.4(6.8)$ \\
\hline \multicolumn{4}{|l|}{ ADHD subtype, $n(\%)^{\mathrm{c}}$} \\
\hline $\begin{array}{l}\text { Predominantly } \\
\text { inattentive }\end{array}$ & $23(20.7)$ & $16(14.5)$ & $14(12.7)$ \\
\hline $\begin{array}{l}\text { Predominantly } \\
\text { hyperactive-impulsive }\end{array}$ & $2(1.8)$ & $7(6.4)$ & $1(0.9)$ \\
\hline Combined & $86(77.5)$ & $87(79.1)$ & $95(86.4)$ \\
\hline \multicolumn{4}{|l|}{$\begin{array}{l}\text { Concomitant psychiatric } \\
\text { diagnosis, } n(\%)^{\mathrm{d}}\end{array}$} \\
\hline Any & $19(17.1)$ & $20(18.2)$ & $29(26.1)$ \\
\hline $\begin{array}{l}\text { Oppositional defiant } \\
\text { disorder }\end{array}$ & $8(7.2)$ & $8(7.3)$ & $10(9.0)$ \\
\hline
\end{tabular}

${ }^{a}$ Demographic and baseline characteristics have previously been reported in detail [13]

b Five patients had no baseline ADHD-RS-IV total score

c One patient in the OROS-MPH group was not evaluated for ADHD subtype. Percentages are based on the number of patients in each treatment group

${ }^{\mathrm{d}}$ Patients with at least one ongoing definite psychiatric diagnosis based on the Kiddie Schedule for Affective Disorders and Schizophrenia for school age children-present and lifetime diagnostic interview. A patient could have more than one diagnosis

$A D H D$ attention-deficit hyperactivity disorder; $A D H D-R S-I V$ ADHD Rating Scale IV, BMI body mass index, $L D X$ lisdexamfetamine dimesylate, OROS-MPH osmotic-release oral system methylphenidate, $S D$ standard deviation

$10.7 \%$ (4.7-16.6) for placebo and $55.9 \%$ (46.2-65.5) for OROS-MPH. The difference (active drug minus placebo) in the percentage of responders was statistically significant for lisdexamfetamine $(p<0.001$; NNT 2$)$ and OROS-MPH $(p<0.001$; NNT 3) (Table 2).

Responders were also defined a posteriori as those achieving an ADHD-RS-IV total score less than or equal to the mean for their age. At endpoint, the percentage of patients $(95 \% \mathrm{CI})$ meeting this second responder criterion was $65.0 \%(55.7-74.3)$ for lisdexamfetamine, $14.4 \%$ (7.7-21.2) for placebo and $51.0 \%$ (41.4-60.6) for OROSMPH. The difference (active drug minus placebo) in the percentage of responders was statistically significant for lisdexamfetamine $(p<0.001$; NNT 2$)$ and OROS-MPH $(p<0.001$; NNT 3) (Table 2).

The post hoc analysis showed a statistically significant difference between lisdexamfetamine and OROS-MPH, in favour of lisdexamfetamine, in the LS mean change in 
Table 2 Summary of efficacy outcomes for lisdexamfetamine dimesylate and osmotic-release oral system methylphenidate in children and adolescents with attention-deficit hyperactivity disorder (full analysis set)

\begin{tabular}{|c|c|c|c|}
\hline & $\begin{array}{l}\text { LDX } \\
\text { minus } \\
\text { placebo }\end{array}$ & $\begin{array}{l}\text { OROS-MPH } \\
\text { minus } \\
\text { placebo }\end{array}$ & $\begin{array}{l}\text { LDX minus } \\
\text { OROS- } \\
\text { MPH }\end{array}$ \\
\hline \multicolumn{4}{|l|}{ ADHD-RS-IV ${ }^{\mathrm{a}}$} \\
\hline $\begin{array}{l}\text { Difference in LS mean } \\
\text { change in ADHD-RS-IV } \\
\text { total score from baseline } \\
\text { to endpoint }\end{array}$ & -18.6 & -13.0 & -5.6 \\
\hline $95 \% \mathrm{CI}$ & $\begin{array}{c}-21.5 \text { to } \\
-15.7\end{array}$ & $\begin{array}{c}-15.9 \text { to } \\
-10.2\end{array}$ & $\begin{array}{l}-8.4 \text { to } \\
-2.7\end{array}$ \\
\hline$p$ value & $<0.001$ & $<0.001$ & $<0.001$ \\
\hline Effect size & 1.80 & 1.26 & 0.54 \\
\hline \multicolumn{4}{|l|}{ CGI-I } \\
\hline $\begin{array}{l}\text { Difference in percentage } \\
\text { of patients 'improved' } \\
\text { at endpoint }(\%)^{\mathrm{b}}\end{array}$ & 63.6 & 46.2 & 17.4 \\
\hline $95 \%$ CI & $53.0-74.1$ & $34.6-57.7$ & $5.0-29.8$ \\
\hline$p$ value & $<0.001$ & $<0.001$ & $<0.05$ \\
\hline NNT & 2 & 3 & 6 \\
\hline \multicolumn{4}{|c|}{$\begin{array}{l}\text { Responders ( } \geq 30 \% \text { reduction from baseline in ADHD-RS-IV } \\
\text { total score and CGI-I of } 1 \text { or } 2)\end{array}$} \\
\hline $\begin{array}{l}\text { Difference in percentage } \\
\text { of responders at } \\
\text { endpoint }(\%)\end{array}$ & 63.5 & 45.2 & 18.3 \\
\hline $95 \%$ CI & $53.0-74.1$ & $33.9-56.5$ & $5.4-31.3$ \\
\hline$p$ value & $<0.001$ & $<0.001$ & $<0.05$ \\
\hline NNT & 2 & 3 & 6 \\
\hline \multicolumn{4}{|c|}{ Responders (ADHD-RS-IV total score $\leq$ mean for age) ${ }^{\text {c }}$} \\
\hline $\begin{array}{l}\text { Difference in percentage } \\
\text { of responders at } \\
\text { endpoint }(\%)\end{array}$ & 50.6 & 36.5 & 14.0 \\
\hline $95 \%$ CI & $39.0-62.1$ & $24.8-48.3$ & $0.6-27.4$ \\
\hline$p$ value & $<0.001$ & $<0.001$ & 0.050 \\
\hline NNT & 2 & 3 & 8 \\
\hline
\end{tabular}

$p$ values are based on the difference between active drug and placebo (predefined comparison) and the difference between LDX and OROSMPH (post hoc comparison). Data are provided for the full analysis set: LDX $(n=104)$; placebo $(n=106)$; OROS-MPH $(n=107)$. All percentages are based on the number of patients with data at that visit in each treatment group. Endpoint was the last on-treatment, postbaseline visit with a non-missing assessment

${ }^{a}$ A decrease from baseline in the ADHD-RS-IV total score indicates an improvement in ADHD symptoms

b Improvement was defined as a CGI-I score of 1 (very much improved) or 2 (much improved)

${ }^{c}$ Responder analysis based on normative data

$A D H D$ attention-deficit hyperactivity disorder; $A D H D-R S-I V$ ADHD Rating Scale IV, CGI-I Clinical Global Impressions-Improvement, $C I$ confidence interval, $L D X$ lisdexamfetamine dimesylate, $L S$ least squares, $N N T$ number needed to treat, $O R O S-M P H$ osmotic-release oral system methylphenidate
ADHD-RS-IV total score from baseline to endpoint $(p<0.001$; effect size 0.54$)$, in the percentage of patients with a CGI-I score of 1 or 2 at endpoint ( $p<0.05$; NNT 6) and in the percentage of patients achieving at least a $30 \%$ reduction from baseline in ADHD-RS-IV total score and a CGI-I score of 1 or 2 at endpoint $(p<0.05$; NNT 6$)$ (Table 2). At endpoint, the difference between lisdexamfetamine and OROS-MPH in the percentage of patients with an ADHD-RS-IV total score less than or equal to the mean for their age was not statistically significant ( $p=0.050$; Table 2).

\subsection{Safety Outcomes}

Safety outcomes have been reported in detail previously [13]. Most patients in the safety population reported one or more TEAEs (Table 3). Of the TEAEs reported in at least $10 \%$ of patients in any treatment group, those that occurred at a numerically greater frequency in the lisdexamfetamine group than in the OROS-MPH group were decreased appetite, insomnia, decreased weight, nausea and anorexia; headache and nasopharyngitis were more frequently reported in the OROS-MPH group than in the lisdexamfetamine group (Table 3). The proportion of patients reporting serious adverse events was low across all treatment groups (lisdexamfetamine $2.7 \%$; placebo $2.7 \%$; OROS-MPH $1.8 \%$ ). Few patients experienced TEAEs leading to discontinuation of study drug (lisdexamfetamine $4.5 \%$; placebo $3.6 \%$; OROS-MPH $1.8 \%$ ).

Patients treated with lisdexamfetamine and OROS-MPH reported modest increases from baseline to endpoint in mean (SD) pulse rate (lisdexamfetamine +5.5 [13.2] bpm; placebo -0.6 [10.6] bpm; OROS-MPH +3.4 [13.2] bpm), heart rate (lisdexamfetamine +5.7 [15.3] bpm; placebo -1.1 [9.6] bpm; OROS-MPH +5.0 [12.8] bpm), systolic blood pressure (lisdexamfetamine +1.0 [9.8] $\mathrm{mmHg}$; placebo +1.0 [9.6] mmHg; OROS-MPH +0.3 [11.1] mmHg), and diastolic blood pressure (lisdexamfetamine +0.2 [9.6] $\mathrm{mmHg}$; placebo +1.2 [8.7] $\mathrm{mmHg}$; OROS-MPH +1.7 [9.9] mmHg) [13]. Changes in mean (SD) body weight from baseline to endpoint were as follows: lisdexamfetamine -2.1 [1.9] kg; placebo +0.7 [1.0] kg; OROS-MPH -1.3 [1.4] kg) [13]. Of the 47 patients (lisdexamfetamine, $n=35$; OROS-MPH, $n=12$ ) who had a potentially clinically significant decrease in weight at endpoint (defined as $\geq 7 \%$ from baseline), three patients (lisdexamfetamine, $n=2$; OROS-MPH, $n=1$ ) moved from a body mass index (BMI) category of healthy weight low (BMI from 5th to 25th percentile) or healthy weight high (BMI from 25th to 85th percentile) to underweight (BMI less than the 5th percentile). 
Table 3 Treatment emergent adverse effects reported by $\geq 5 \%$ of patients in any treatment group (safety population) ${ }^{\mathrm{a}}$

${ }^{\text {a }}$ Safety outcomes have previously been reported in detail [13]

b TEAEs are presented in order of decreasing frequency in the LDX treatment group

$L D X$ lisdexamfetamine dimesylate, $O R O S-M P H$ osmotic-release oral system methylphenidate, TEAE treatment-emergent adverse event

\begin{tabular}{lccc}
\hline $\begin{array}{l}\text { TEAE, preferred } \\
\text { term, } n(\%)\end{array}$ & LDX $(n=111)$ & Placebo $(n=110)$ & $\begin{array}{l}\text { OROS-MPH } \\
(n=111)\end{array}$ \\
\hline $\begin{array}{l}\text { Any TEAE } \\
\text { TEAEs }(\geq \mathbf{5} \% \text { of patients in any treatment group) }\end{array}$ & $63(57.3)$ & $72(64.9)$ \\
Decreased appetite & $0(25.2)$ & $3(2.7)$ & $17(15.3)$ \\
Headache & $16(14.4)$ & $22(20.0)$ & $22(19.8)$ \\
Insomnia & $16(14.4)$ & 0 & $9(8.1)$ \\
Decreased weight & $15(13.5)$ & 0 & $5(4.5)$ \\
Nausea & $12(10.8)$ & $3(2.7)$ & $8(7.2)$ \\
Anorexia & $12(10.8)$ & $2(1.8)$ & $6(5.4)$ \\
Nasopharyngitis & $8(7.2)$ & $8(7.3)$ & $14(12.6)$ \\
Upper abdominal pain & $8(7.2)$ & $6(5.5)$ & $9(8.1)$ \\
Abdominal pain & $6(5.4)$ & $6(5.5)$ & $4(3.6)$ \\
Sleep disorder & $6(5.4)$ & $1(0.9)$ & $2(1.8)$ \\
Cough & $3(2.7)$ & 0 & $8(7.2)$ \\
Initial insomnia & $3(2.7)$ & $1(0.9)$ & $7(6.3)$ \\
\hline
\end{tabular}

\section{Discussion}

In this post hoc analysis of data from a European, 7-week, phase III study (SPD489-325), children and adolescents treated with lisdexamfetamine showed statistically significantly greater improvements in ADHD-RS-IV total score and CGI-I score from baseline to endpoint than those treated with OROS-MPH. In addition, a greater proportion of patients receiving lisdexamfetamine were categorized as responders at study endpoint than those receiving OROSMPH. These findings suggest that, at the doses tested, lisdexamfetamine produced greater improvements in the symptoms of ADHD in children and adolescents than OROS-MPH.

To date, there has only been one published parallelgroup comparison of stimulant medications for the treatment of individuals with ADHD [17]. In this study, mixed amfetamine salts were found to produce significantly greater improvements in teacher ratings and CGI-I scores than short-acting methylphenidate [17]. Several crossover studies have investigated the comparative efficacies of short-acting methylphenidate- and amfetamine-based stimulants, but no consistent differences have emerged [2]. However, a meta-analysis of randomized controlled trials of both short- and long-acting formulations found that effect sizes for amfetamine-based stimulants were moderately, but statistically significantly, greater than those for methylphenidate [1]. While SPD489-325 was not prospectively designed or powered to compare the clinical profiles of the two active treatment arms, this post hoc analysis has provided the first parallel-group comparison of the efficacy of the long-acting stimulants, lisdexamfetamine and OROS-MPH. Although both treatments produced robust responses, improvements in symptoms were greater for lisdexamfetamine than for OROS-MPH.

In the present study, patients who were randomized to the lisdexamfetamine treatment group received 30,50 or $70 \mathrm{mg} /$ day. OROS-MPH was administered according to European regulations (maximum licensed dose, $54 \mathrm{mg} /$ day). The study included a 3-week dose-optimization period, suggesting that the doses of lisdexamfetamine and OROSMPH were less likely to have influenced their relative efficacy. However, it is notable that a higher proportion of patients was optimized to the highest available dose of OROS-MPH (18 mg/day, $9.9 \% ; 36 \mathrm{mg} /$ day, $19.8 \%$; $54 \mathrm{mg} /$ day, $53.2 \%$ ) than to the highest available dose of lisdexamfetamine (30 mg/day, $18.0 \%$; $50 \mathrm{mg}$ /day, $29.7 \%$; $70 \mathrm{mg} /$ day, $33.3 \%$ ) [13]. Furthermore, the proportion of patients who were discontinued from the study due to lack of efficacy was greater for OROS-MPH than for lisdexamfetamine [13]. Therefore, it is possible that treatment responses were dose limited in more patients receiving OROS-MPH than in those receiving lisdexamfetamine.

There is little evidence to suggest that differences in the baseline patient characteristics contributed to the observed differences in treatment responses to lisdexamfetamine and OROS-MPH. Patients were randomized to receive lisdexamfetamine, placebo or OROS-MPH, and patient demographics and baseline disease characteristics were similar across treatment groups [13]. Although the proportion of patients with the predominantly inattentive subtype was numerically greater for lisdexamfetamine than for OROS-MPH, most patients across all treatment groups had the combined ADHD subtype, and previous analyses revealed that improvements in both the hyperactivity/ impulsivity and the inattention subscale scores of the 
ADHD-RS-IV in response to lisdexamfetamine and OROS-MPH treatment were similar [13]. The proportion of patients with a concomitant, non-exclusionary psychiatric diagnosis was greater for OROS-MPH than for lisdexamfetamine. However, there was a minimal difference in the proportions of patients with oppositional defiant disorder, which has been shown to influence responses to stimulant treatment [18].

Although the mechanisms of action of stimulants in the treatment of ADHD remain to be fully established, it is possible that differences in the pharmacologies of methylphenidate- and amfetamine-based stimulants contributed to the differential treatment responses observed for lisdexamfetamine and OROS-MPH [19]. Differences in formulation and the resulting pharmacokinetic profiles of these long-acting stimulants may also have influenced their therapeutic activity. The pharmacokinetic profile of $d$-amfetamine following administration of lisdexamfetamine is monophasic, sustained and dose proportional [20]. The time to maximum observed plasma concentration $\left(T_{\max }\right)$ and half-life $\left(t_{1 / 2}\right)$ for $d$-amfetamine following administration of lisdexamfetamine $(30,50$ or $70 \mathrm{mg} /$ day $)$ are $3.41-3.58 \mathrm{~h}$ and $8.61-8.90 \mathrm{~h}$, respectively [20]. These pharmacokinetic properties are reflected in the clinical duration of action of lisdexamfetamine, which extends to at least $13 \mathrm{~h}$ post-dose in children and $14 \mathrm{~h}$ post-dose in adults [10, 11]. As the metabolism of lisdexamfetamine occurs mostly in the bloodstream [7], it is unlikely to be affected by variations in gastric $\mathrm{pH}$ or gastrointestinal transit time [9]. The intra- and inter-patient variability in pharmacokinetic parameters is low, reflecting predictable and consistent exposure to $d$-amfetamine following administration of lisdexamfetamine [21]. OROS-MPH uses a mechanical mode of delivery, releasing methylphenidate in a biphasic manner as it transits through the gastrointestinal tract [9]. Approximately $22 \%$ of the overall dose of methylphenidate is immediately released from the drug overcoat of the capsule, providing a rapid onset of clinical efficacy. This is followed by the sustained, osmotically driven release of methylphenidate. In contrast to lisdexamfetamine, alterations in gastrointestinal transit time and first pass metabolism in the liver may have an impact on the delivery of methylphenidate from OROS-MPH to sites of action [9]. Emerging evidence suggests that genetic factors may also influence treatment responses. To date, most pharmacogenetic studies of stimulants for ADHD have focused on genetic variability associated with their potential mechanism of action and have failed to yield consistent, clinically relevant findings [22-24]. It is now also being recognized that genetic variability in carboxylesterase 1A, the principal enzyme responsible for the metabolism of $d$, l-methylphenidate to the inactive metabolite, ritalinic acid, may have an impact on dose requirements [25]. Overall, inter- and intra-patient variability in pharmacokinetic parameters appears to be higher for OROS-MPH than for lisdexamfetamine [9]. Consistent with this, although the therapeutic benefits of OROS-MPH have been shown to last at least $12.5 \mathrm{~h}$ [12], clinical experience suggests that there is considerable variation in the duration of response [6].

In this study, no new safety signals of concern were observed and the safety profiles of lisdexamfetamine and OROS-MPH were similar to the known effects of stimulant medications [26]. However, it is notable that the overall frequency of TEAEs and the proportion of patients who were discontinued from the study due to TEAEs was numerically greater for lisdexamfetamine than for OROSMPH. In addition, certain TEAEs, including decreased appetite, insomnia, decreased weight, nausea and anorexia, occurred more frequently in patients treated with lisdexamfetamine than in those who received OROS-MPH; none of these TEAEs were serious [13]. The modest mean increases from baseline in heart rate, pulse rate, and systolic and diastolic blood pressure in patients receiving lisdexamfetamine and OROS-MPH were also consistent with the known safety profiles of stimulant medications [26]. Finally, the decrease in mean weight was numerically greater in the lisdexamfetamine treatment group than in the OROS-MPH group. However, most patients in both active treatment groups remained within their baseline BMI category and few participants had potentially clinically important weight changes that resulted in a shift to the underweight BMI category. Overall, decisions regarding the choice of ADHD medication for individual patients should take into account the balance between the benefits and risks associated with each treatment.

\section{Conclusions}

In this post hoc analysis of data from a European, randomized, phase III study, children and adolescents with ADHD who were treated with lisdexamfetamine showed statistically significantly greater improvements in ADHDRS-IV total score and CGI-I score from baseline to endpoint than those treated with OROS-MPH. This suggests that, at the doses tested, patients treated with lisdexamfetamine showed greater improvements in symptoms of ADHD than those who received OROS-MPH. The results of ongoing parallel-group clinical studies (ClinicalTrials.gov: NCT01552915 and NCT01552902) [27, 28] will provide definitive evidence of the comparative therapeutic efficacy of lisdexamfetamine and OROS-MPH. Meanwhile, the results of the present post hoc analysis support lisdexamfetamine as a valuable treatment option for the management of children and adolescents with ADHD. 
Funding This study was supported by funding from Shire Development LLC.

Author contributions T Banaschewski, DR Coghill, M Johnson, M Lecendreux, C Soutullo and A Zuddas were principal investigators in this clinical study. C Anderson, R Civil, N Higgins and LA Squires contributed to the study design. R Bloomfield was responsible for the statistical analysis. All authors were involved in discussion and interpretation of the data, critically revised the article and approved the manuscript before submission.

Acknowledgments The authors thank the patients and investigators who took part in this study. They also thank Dr Eric Southam and Dr Elizabeth Gandhi of Oxford PharmaGenesis ${ }^{\mathrm{TM}}$ Ltd who provided editorial assistance funded by Shire Development LLC, including collating the comments of the authors and editing the manuscript for submission.

Conflicts of interest C Anderson, R Bloomfield, R Civil, N Higgins and L Squires are employees of Shire and own stock/stock options. The following authors have received compensation for serving as consultants or speakers, or they or the institutions they work for have received research support or royalties from the companies or organizations indicated: DR Coghill (Flynn Pharma, Janssen-Cilag, Lilly, Medice, Novartis, Otsuka, Oxford University Press, Pfizer, ScheringPlough, Shire, UCB, Vifor Pharma); T Banaschewski (Bristol-Myers Squibb, Desitin, Develco Pharma, Janssen McNeil, Lilly, Medice, Novartis, Shire, UCB, Vifor Pharma); M Lecendreux (Shire, UCB, Vifor Pharma); C Soutullo (Abbott, Alicia Koplowitz Foundation, AstraZeneca, Bristol-Myers Squibb, Institute of Health Carlos III [FIS]: Redes Temáticas de Investigación Cooperativa, Doyma, Editorial Médica Panamericana, Eli Lilly, EUNSA [University of Navarra Press], European Interdisciplinary Network ADHD Quality Assurance, Euro RSCG Life Medea, GlaxoSmithKline, Gobierno de Navarra, Grupo Aula Médica, Grupo Correo, Janssen, Medice/Juste, Novartis, Otsuka, Pfizer, University of Navarra Research Projects [PIUNA], Rubiò, Shire, Sociedad Vasco-Navarra Psiquiatría, Solvay, Stanley Medical Research Institute - National Alliance on Mental Illness, Wolters Kluwer); M Johnson (Janssen, Lilly, Novartis, Shire, Vifor Pharma, Lundgrens Research Fund); A Zuddas (AstraZeneca, Bristol-Myers Squibb/Otsuka, Lilly, Lundbeck, Shire, Vifor Pharma).

Open Access This article is distributed under the terms of the Creative Commons Attribution Noncommercial License which permits any noncommercial use, distribution, and reproduction in any medium, provided the original author(s) and the source are credited.

\section{References}

1. Faraone SV, Buitelaar J. Comparing the efficacy of stimulants for ADHD in children and adolescents using meta-analysis. Eur Child Adolesc Psychiatry. 2010;19(4):353-64.

2. Hodgkins P, Shaw M, Coghill D, Hechtman L. Amfetamine and methylphenidate medications for attention-deficit/hyperactivity disorder: complementary treatment options. Eur Child Adolesc Psychiatry. 2012;21(9):477-92.

3. Polanczyk G, de Lima MS, Horta BL, Biederman J, Rohde LA. The worldwide prevalence of ADHD: a systematic review and metaregression analysis. Am J Psychiatry. 2007;164(6):942-8.

4. American Psychiatric Association. Diagnostic and statistical manual of mental disorders, 4th ed, text revision. Washington, DC: American Psychiatric Association; 2000: 85-93.
5. Antshel KM, Hargrave TM, Simonescu M, Kaul P, Hendricks K, Faraone SV. Advances in understanding and treating ADHD. BMC Med. 2011;9:72.

6. Banaschewski T, Coghill D, Santosh P, Zuddas A, Asherson P, Buitelaar J, et al. Long-acting medications for the hyperkinetic disorders: a systematic review and European treatment guideline. Eur Child Adolesc Psychiatry. 2006;15(8):476-95.

7. Pennick M. Absorption of lisdexamfetamine dimesylate and its enzymatic conversion to $d$-amphetamine. Neuropsychiatr Dis Treat. 2010;6:317-27.

8. Goodman DW. Lisdexamfetamine dimesylate: the first prodrug stimulant. Psychiatry (Edgmont). 2007;4(8):39-45.

9. Ermer JC, Adeyi BA, Pucci ML. Pharmacokinetic variability of long-acting stimulants in the treatment of children and adults with attention-deficit hyperactivity disorder. CNS Drugs. 2010;24(12): 1009-25.

10. Wigal SB, Kollins SH, Childress AC, Squires L. A 13-hour laboratory school study of lisdexamfetamine dimesylate in school-aged children with attention-deficit/hyperactivity disorder. Child Adolesc Psychiatry Ment Health. 2009;3(1):17.

11. Wigal T, Brams M, Gasior M, Gao J, Squires L, Giblin J. Randomized, double-blind, placebo-controlled, crossover study of the efficacy and safety of lisdexamfetamine dimesylate in adults with attention-deficit/hyperactivity disorder: novel findings using a simulated adult workplace environment design. Behav Brain Funct. 2010;6:34.

12. Armstrong RB, Damaraju CV, Ascher S, Schwarzman L, O'Neill J, Starr HL. Time course of treatment effect of OROS(R) methylphenidate in children with ADHD. J Atten Disord. 2011;6:34.

13. Coghill D, Banaschewski T, Lecendreux M, Soutullo C, Johnson M, Zuddas A, et al. European, randomized, phase 3 study of lisdexamfetamine dimesylate in children and adolescents with attention-deficit/hyperactivity disorder. Eur Neuropsychopharmacol. 14 Jan 2013. http://dx.doi.org/10.1016/j.euroneuro.2012. 11.012 .

14. European Medicines Agency. Guideline on the clinical investigation of medicinal products for the treatment of attention deficit hyperactivity disorder (ADHD) [online]. Available from URL: http://www.ema.europa.eu/docs/en_GB/document_library/ Scientific_guideline/2010/08/WC500095686.pdf. Accessed 16 May 2013.

15. Concerta XL 18-36 mg. Summary of Product Characteristics [online]. Available from URL: http://www.medicines.org.uk/ $\mathrm{EMC} /$ medicine/8382/SPC/Concerta $+\mathrm{XL}+18+\mathrm{mg}+-+36+\mathrm{mg}+$ prolonged+release+tablets. Accessed 27 Oct 2012.

16. Findling RL, Adeyi B, Chen G, Dirks B, Babcock T, Scheckner B, Lasser R, Pucci ML, Abdullah HI, McGough JJ. Clinical response and symptomatic remission in children treated with lisdexamfetamine dimesylate for attention-deficit/hyperactivity disorder. CNS Spectr. 2010;15(9):559.

17. Pliszka SR, Browne RG, Olvera RL, Wynne SK. A double-blind, placebo-controlled study of Adderall and methylphenidate in the treatment of attention-deficit/hyperactivity disorder. J Am Acad Child Adolesc Psychiatry. 2000;39(5):619-26.

18. Goez H, Back-Bennet O, Zelnik N. Differential stimulant response on attention in children with comorbid anxiety and oppositional defiant disorder. J Child Neurol. 2007;22(5):538-42.

19. Hodgkins P, Shaw M, McCarthy S, Sallee FR. The pharmacology and clinical outcomes of amphetamines to treat ADHD: does composition matter? CNS Drugs. 2012;26(3):245-68.

20. Boellner SW, Stark JG, Krishnan S, Zhang Y. Pharmacokinetics of lisdexamfetamine dimesylate and its active metabolite, d-amphetamine, with increasing oral doses of lisdexamfetamine dimesylate in children with attention-deficit/hyperactivity disorder: a single-dose, randomized, open-label, crossover study. Clin Ther. 2010;32(2):252-64. 
21. Biederman J, Boellner SW, Childress A, Lopez FA, Krishnan S, Zhang Y. Lisdexamfetamine dimesylate and mixed amphetamine salts extended-release in children with ADHD: a double-blind, placebo-controlled, crossover analog classroom study. Biol Psychiatry. 2007;62(9):970-6.

22. Contini V, Rovaris DL, Victor MM, Grevet EH, Rohde LA, Bau $\mathrm{CH}$. Pharmacogenetics of response to methylphenidate in adult patients with attention-deficit/hyperactivity disorder (ADHD): a systematic review. Eur Neuropsychopharmacol. Epub 16 June 2012.

23. Froehlich TE, Epstein JN, Nick TG, Melguizo Castro MS, Stein MA, Brinkman WB, et al. Pharmacogenetic predictors of methylphenidate dose-response in attention-deficit/hyperactivity disorder. J Am Acad Child Adolesc Psychiatry. 2011; 50(11): 1129-39 e2.
24. Froehlich TE, McGough JJ, Stein MA. Progress and promise of attention-deficit hyperactivity disorder pharmacogenetics. CNS Drugs. 2010;24(2):99-117.

25. Nemoda Z, Angyal N, Tarnok Z, Gadoros J, Sasvari-Szekely M. Carboxylesterase 1 gene polymorphism and methylphenidate response in ADHD. Neuropharmacology. 2009;57(7-8):731-3.

26. May DE, Kratochvil CJ. Attention-deficit hyperactivity disorder: recent advances in paediatric pharmacotherapy. Drugs. 2010;70(1): $15-40$.

27. US National Institutes of Health. ClinicalTrials.gov [online]. Available from URL: http://clinicaltrialsgov/show/NCT01552915. Accessed 14 Feb 2013.

28. US National Institutes of Health. ClinicalTrials.gov [online]. Available from URL: http://clinicaltrialsgov/show/NCT01552902. Accessed 14 Feb 2013. 